CERN-PPE/95-62

23.03.1995

\title{
PERFORMANCE AND CALIBRATION OF THE CHORUS SCINTILLATING FIBER TRACKER AND OPTO-ELECTRONICS READOUT SYSTEM
}

P. Annis ${ }^{a}$, S. Aoki ${ }^{b}$, G. Brooijmans ${ }^{c, d}$, J. Brunner ${ }^{e}$, M. de Jong ${ }^{f}$, J.-P. Fabre ${ }^{e}$, R. Ferreira ${ }^{e}$, W. Flegel ${ }^{e}$, D. Frekers ${ }^{g}$, G. Grégoire $^{c}$, M. Gruwée ${ }^{a, l}$, J. Hérin $^{c}$, K. Hoepfner ${ }^{h}$, M. Kobayashi ${ }^{i}$, J. Konijn $^{f}$, V. Lemaitre $^{c}$, P. Lendermann ${ }^{h}$, D. Macina ${ }^{e}$, R. Meijer Drees ${ }^{e}$, H. Meinhard ${ }^{e}$, L. Michel ${ }^{c, d}$, C. Mommaert $^{a, d}$, K. Nakamura ${ }^{j}$, M. Nakamura ${ }^{i}$, T. Nakano ${ }^{i}$, K. Niwa ${ }^{i}$, E. Niu ${ }^{k}$, J. Panman $^{e}$, F. Riccardi ${ }^{e}$, D. Rondeshagen ${ }^{g}$, O. Sato ${ }^{i}$, G. Stefanini ${ }^{e}$, M. Vander Donckt ${ }^{a, m}$, P. Vilain $^{a, l}$, G. Wilquet ${ }^{a, l}$, K. Winter ${ }^{e}$, H.T. Wong ${ }^{e}$.

${ }^{a}$ IIHE ULB-VUB, Brussels, Belgium

${ }^{b}$ Kobe University, Kobe, Japan

${ }^{c}$ Université Catholique de Louvain, Louvain-la-Neuve, Belgium

${ }^{d}$ Inter-University Institute for Nuclear Science, Belgium

${ }^{e}$ CERN, Geneva, Switzerland

$f$ NIKHEF, Amsterdam, The Netherlands

${ }^{g}$ Westfälische Wilhelms-Universität, Münster, Germany

${ }^{h}$ Humboldt-Universität, Berlin, Germany

$i$ Nagoya University, Nagoya, Japan

${ }^{j}$ Osaka City University, Osaka, Japan

${ }^{k}$ Toho University, Funabashi, Japan

${ }^{l}$ Fonds National pour la Recherche Scientifique, Belgium

${ }^{m}$ Fonds pour la Recherche scientifique, l'Industrie et L'Agriculture, Belgium

\begin{abstract}
An essential component of the CERN WA95/CHORUS experiment is a scintillating fiber tracker system for precise track reconstruction of particles. The tracker design, its opto-electronics readout and calibration system are discussed. Performances of the detector are presented.
\end{abstract}

presented by Dirk Rondeshagen to

The 7th Wire Chamber Conference, Vienna 1995.

(Submitted to Nucl. Inst. \& Meth.) 


\section{Introduction}

Neutrino masses and mixings, if they exist, will have profound implications in particle physics and cosmology [1]. The investigations of these fundamental neutrino properties remain one of the great challenges in experimental particle physics. The objective of the CERN WA95/CHORUS experiment [2] is to search for neutrino oscillations in the $\nu_{\mu} \rightarrow \nu_{\tau}$ appearance channel with the CERN SPS wide band neutrino beam. The CHORUS detector is fully operational and physics data taking has started in May 1994.

The schematic diagram of the CHORUS detector is depicted in Figure 1. A total of $800 \mathrm{~kg}$ of nuclear emulsion is used as target material. The signature for $\nu_{\mu} \rightarrow \nu_{\tau}$ oscillation is the explicit vertex detection of the charged current interaction $\nu_{\tau} N \rightarrow \tau^{-} X$ and the subsequent decay kink of the short-lived $\tau^{-}$to its daughters, shown schematically in Figure 2. The experiment is expected to achieve the sensitivity of $\sin ^{2} 2 \theta_{\mu \tau} \sim 3 \times 10^{-4}$ at large $\Delta m^{2}$, an interesting parameter space for the hypothesis of $\nu_{\tau}$ as a Dark Matter candidate [3].

The experimental set-up consists of the emulsion target, a scintillating fiber tracker system, an air core magnet, a calorimeter based on spaghetti technique, and a muon spectrometer. The emulsion target, tracker system and magnet are placed in a "cold box" stabilized at $5^{\circ} \mathrm{C}$, in order to reduce fading of the emulsion as well as the ageing of the scintillating fibers.

Nuclear emulsion offers a superb spatial resolution of $1 \mu \mathrm{m}$, and is therefore ideal for the detection of short-lived particles. The fiber tracker system provides accurate trajectory predictions back to the emulsion target, as well as a good two-track resolution crucial for event reconstruction. Consequently, the required emulsion scanning time can be greatly reduced.

\section{The CHORUS Fiber Tracker System}

\subsection{Detector Design}

The CHORUS tracker system is based on plastic scintillating fibers of $2.3 \mathrm{~m}$ in length, and $500 \mu \mathrm{m}$ in diameter. Seven layers of these fibers are glued to form a ribbon in a "staggered" geometry as shown in Figure 3. The far end of the ribbons is sputtered with aluminium which gives a reflectivity of about $80 \%$. Detailed descriptions of the tracker design and geometry [4], the fiber-winding machine [5], and the mass production of multi-layer fiber ribbons [6] have been reported in other publications.

The CHORUS tracker system consists of two components : 1) the target tracker, shown schematically in Figure 2, allows high precision tracking which is crucial for the accurate predictions of the scanning region in the interface emulsion sheets (shown as CS1 and CS2 in Figure 2) which are changed once every three weeks. Fully automatic 
microscopes controlled by computers are used to scan these sheets, and subsequently to locate the interaction vertices in the emulsion target; and 2) the magnet tracker, placed upstream and downstream of the air-core magnet, allows the measurement of charge and momentum of traversing particles up to $\sim 10 \mathrm{GeV} / \mathrm{c}$.

The entire tracker system comprises more than one million fibers with a total length of about $2500 \mathrm{~km}$. The readout ends of the fiber ribbons are bundled together and coupled to a total of 58 opto-electronics readout chains, each of which consists of four image intensifiers and a CCD camera in series, as depicted schematically in Figure 4. Detailed descriptions of the characteristics of the image intensifiers [7] and CCD cameras [8] are documented in the references listed.

\subsection{Calibration System}

As shown schematically in Figure 5, a total of 16 fiber ribbons are bundled together at the readout end, with spacers separating adjacent ribbons. The spacers have a thickness of $200 \mu \mathrm{m}$, except five which are $500 \mu \mathrm{m}$ in thickness, and contain 45 clear fibers (9 per spacer) of $127 \mu \mathrm{m}$ in diameter. These "fiducial" fibers are grouped together and coupled through a fiber optic cable to an LED pulser module. Details of the construction and tests of the calibration system can be found in [9]. Calibration and optimization of the opto-electronics readout chains are achieved with this system.

The exact dimension of the fiber bundles and the positions of the fiducial fibers were measured with photographic contact prints before the opto-electronic readout chains were mounted on to the fiber bundles. The geometry is shown in Figure 6a. Electrostatic distortion from the image intensifiers turns this input pattern into one shown in Figure 6b. By measuring the positions of the fiducial fibers and comparing them with their contact print co-ordinates, a fit for the distortion correction can be performed using the expression $R_{C C D}=A R_{\text {in }}\left(1+B\left(R_{\text {in }} / R_{0}\right)^{\alpha}\right)$, where $R_{C C D}$ and $R_{\text {in }}$ represent the radial positions of the fiducial fibers on the CCD and on the input window from the optical centers, respectively, and $R_{0}=5 \mathrm{~cm}$ is the radius of the input window. The fitted parameter $\mathrm{A}(\sim 0.1)$ describes the demagnification of the opto-electronics readout, whereas $\mathrm{B}(\sim 0.25)$ and $\alpha(\sim 2.7)$ parametrize the distortion. The residuals of the fit have a $\sigma \sim 110 \mu \mathrm{m}$ at the input window.

The fiducial fiber system is also used for optimization of the operation parameters for the opto-electronics readout. The LED intensity is adjusted to produce single photoelectrons at the first photocathode of the readout chain. The optimal operation parameters, such as the image intensifier focus and zooming voltages as well as the Micro Channel Plate (MCP) voltage and gate time, can be determined by studying the response of the image intensifiers and the CCD for various values of these parameters.

By comparing with the measurements using a photo-multiplier with known quantum efficiency, the quantum efficiency of the opto-electronics chains is determined to 
be about $18 \%$. There are two aspects which characterize the spatial resolution of the readout system. The "spot size", (the width of the charge distribution induced on the CCD from a single photo-electron produced in the first image intensifier) is due to the intrinsic resolution of the opto-electronics readout chain, whereas the "spot displacement" (the deviation of the center of gravity of a cluster from its mean position) is caused mainly by the focusing of the first image intensifier. The standard deviation for the spot size and displacement, based on the measured fiducial width, are $136 \mu \mathrm{m}$ and $89 \mu \mathrm{m}$ at the input window, respectively.

\subsection{Detector Hardware Performance}

Muons with energy of about $25 \mathrm{GeV}$ are also produced from neutrino interactions upstream to the detector. These muons were used for measuring the properties of the fiber trackers, as well as for achieving precise alignment among various tracker planes. The signatures recorded by the CCD when such beam muons (or other minimum ionizing particles) traverse a $3 \mathrm{~mm}$ fiber ribbon are depicted in Figure 7 . The crosses superimposed on the display are the "hits" as identified by software "clustering" algorithms [10], corresponding to detected single photo-electrons.

The ribbon hit density $(\mathrm{Y})$ as a function of distance $(\mathrm{x})$ from the readout is shown in Figure 8 . The distribution can be described by

$$
\mathrm{Y}=\frac{\mathrm{A}}{1+\mathrm{r}}\left[\exp \left(-\frac{(\mathrm{x}-\mathrm{L})}{\lambda}\right)+\mathrm{r} \times \exp \left(-\frac{(\mathrm{L}-\mathrm{x})}{\lambda}\right)\right]
$$

In the expression, $\mathrm{L}=230 \mathrm{~cm}$ is the length of the fiber, and $\mathrm{r}=0.8$ is the mirror reflectivity at the far end of the fiber. The fitted quantity A denotes the hit density at $\mathrm{x}=\mathrm{L}(\sim 5$ hits per ribbon), whereas $\lambda \sim 200 \mathrm{~cm}$ is the "bare" attenuation length of the scintillating fibers. The "effective" attenuation length can be evaluated by fitting the distribution to a single exponential, and is measured to be $\lambda_{\text {eff }} \sim 600 \mathrm{~cm}$. From Figure 8 , it can be seen that the hit density is about 5 and 7 hits/ribbon at the far $(230 \mathrm{~cm})$ and near $(70 \mathrm{~cm})$ ends of the detector, respectively.

The tracker detector inefficiency per ribbon (the probability of a minimum ionizing particle passing through without producing any signals recorded by the CCD) can also be evaluated using the beam muon data, and is measured to be about $2 \times 10^{-3}$.

The tracking performance of the fiber trackers depends on the precise knowledge of the fiber positions within a ribbon, and of the alignment of different tracker planes relative to each other and to the interface emulsion sheets. To date, the achieved hits residual (deviation of hits from the best fitted trajectory) has a $\sigma \sim 350 \mu \mathrm{m}$, whereas the prediction accuracy (deviation of predicted positions on the interface emulsion sheets from the found tracks) has a $\sigma \sim 250 \mu \mathrm{m}$. Improvement of these values are expected from further work on inter-tracker and tracker-emulsion alignment and calibration. 


\section{Conclusion}

The CHORUS Tracker has been successfully constructed and commissioned on schedule. It is the largest tracking system based on scintillating fibers built so far. The expected hardware performance in terms of hits density, fiber attenuation length and readout spatial resolution have been achieved. Correction on the electrostatic distortion of the opto-electronics readout has been performed to the required accuracy. Tracking performance from hit residuals and prediction accuracy are satisfactory with further improvement foreseen.

The emphasis of our work is on the precision alignment and calibration of the tracker planes and the interface emulsion sheets, as well as on the event reconstruction and momentum measurement of the through-going particles from the recorded neutrino events.

\section{Acknowledgement}

The authors would like to thank D. Bourillot, G. Carnevale, J. Dupraz, J. Dupont, H. von Heynitz, P. Nappey, R. Pintus, S. Reynaud, and G. Van Beek for their invaluable technical support. 


\section{References}

1. For an overview on the subject of neutrino physics, see, for example, "Neutrino Physics", ed. K. Winter, Cambridge University Press (1991).

2. CHORUS Proposal, N. Armenise et al., CERN-SPSC/90-42, (1990); M. de Jong et al., CERN-PPE/93-131 (1993).

3. H. Harari, Phys. Lett. B216, 413 (1989);

J. Ellis, J.L. Lopez, and D.V. Nanopoulos, Phys. Lett. B292, 189 (1992);

H. Fritzsch and D. Holtmannspötter, Phys. Lett. B338, 290 (1994).

4. S. Aoki et al., Nucl. Instrum. Methods A 344, 143 (1994).

5. T. Nakano et al., in Proc. of IEEE 1991 Nuclear Science Symp., Santa Fe (1991).

6. T. Nakano et al., in Proc. of Scintillating Fiber Symposium 1993, University of Notre Dame (1993).

7. M. Gruwé, Ph.D. Thesis, Université Libre de Bruxelles (1994).

8. C. Mommaert, Ph.D. Thesis, Vrije Universiteit Brussel (1995).

9. D. Rondeshagen, Dipl. Thesis, Westfälische Wilhelms-Universität (1994).

10. P. Lendermann, Ph.D. Thesis, Humboldt-Universität (1994). 


\section{Figure Captions}

1. Schematic diagram of the CHORUS detector.

2. Schematic diagram showing the geometry of the emulsion target, the interface emulsion sheets (CS1 and CS2) and the target trackers. Displayed in the target is the typical signature of a $\nu_{\tau}$ charged-current interaction.

3. Cross section of a scintillating fiber ribbon, showing its "staggered" geometry.

4. The configuration of the opto-electronics readout system.

5. Schematic diagram of a fiber bundle and calibration fiducial fibers to be coupled to the readout system.

6. A typical fiber ribbon bundle as measured in the (a) input, and (b) output of the readout system, showing the effects due to electrostatic distortion from the image intensifiers. The units in (b) are in CCD pixel number, translating to $\mathrm{X}=145 \mu \mathrm{m}$ and $\mathrm{Y}=209 \mu \mathrm{m}$ per unit in detector space. Small black dots denote the ribbon boundary.

7. The typical signature of a traversing muon through a fiber ribbon, as recorded by the CCD. Superimposed as crosses are the "hits" as identified by software. Units are in CCD pixel number, as explained in Figure $6 \mathrm{~b}$.

8. The distribution of ribbon hit density as function of distance from readout photocathode. 


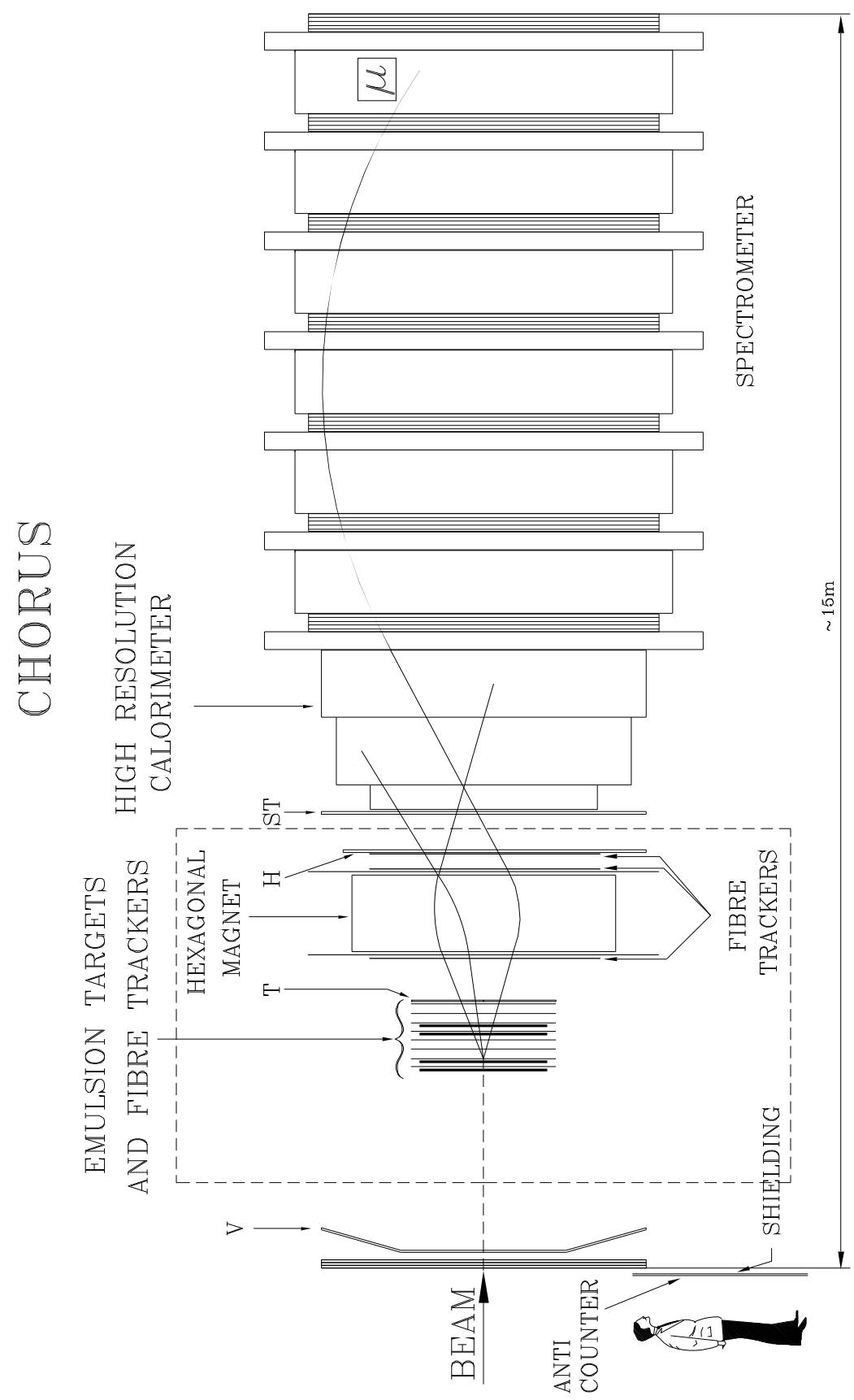

Figure 1 


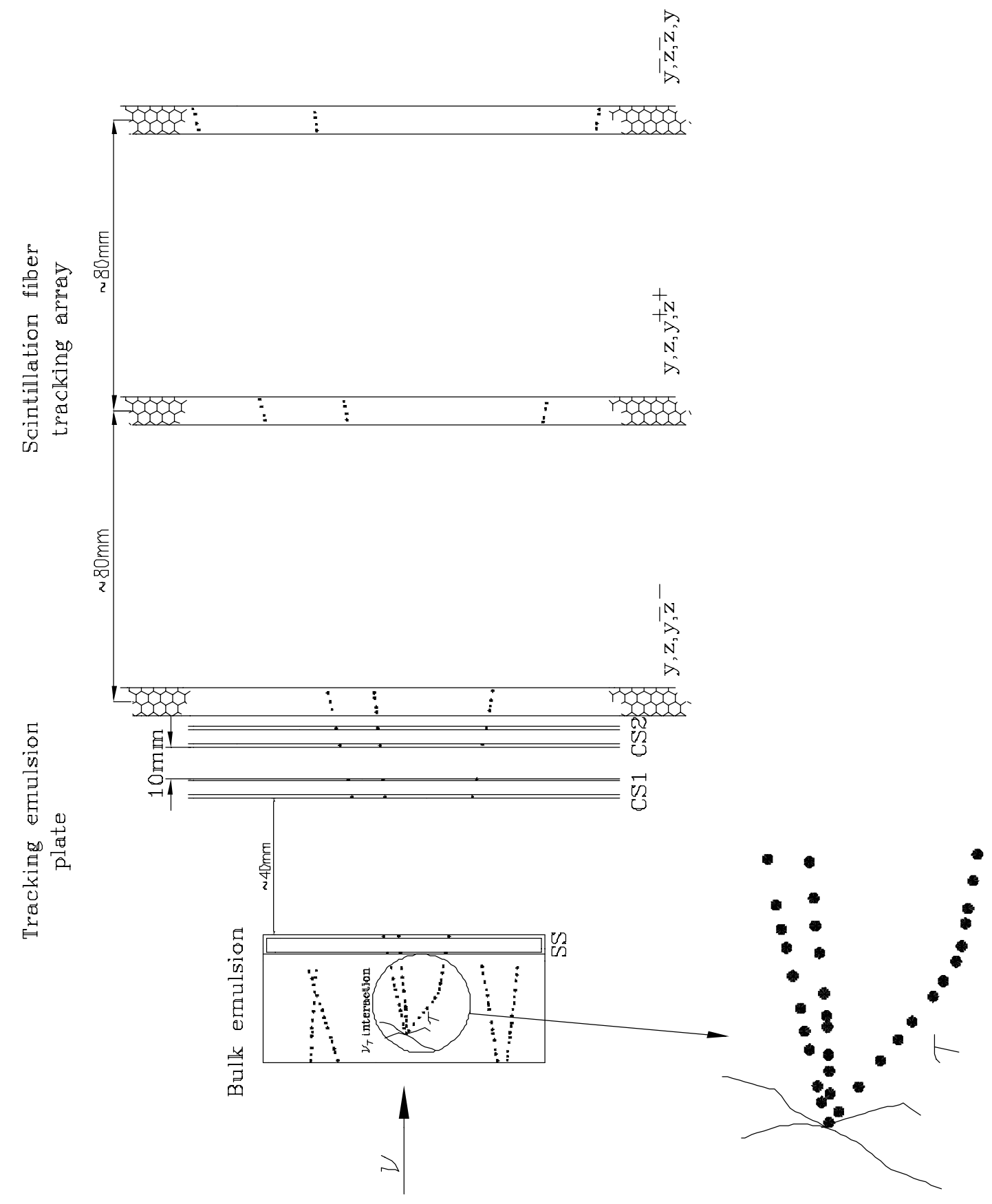

Figure 2 


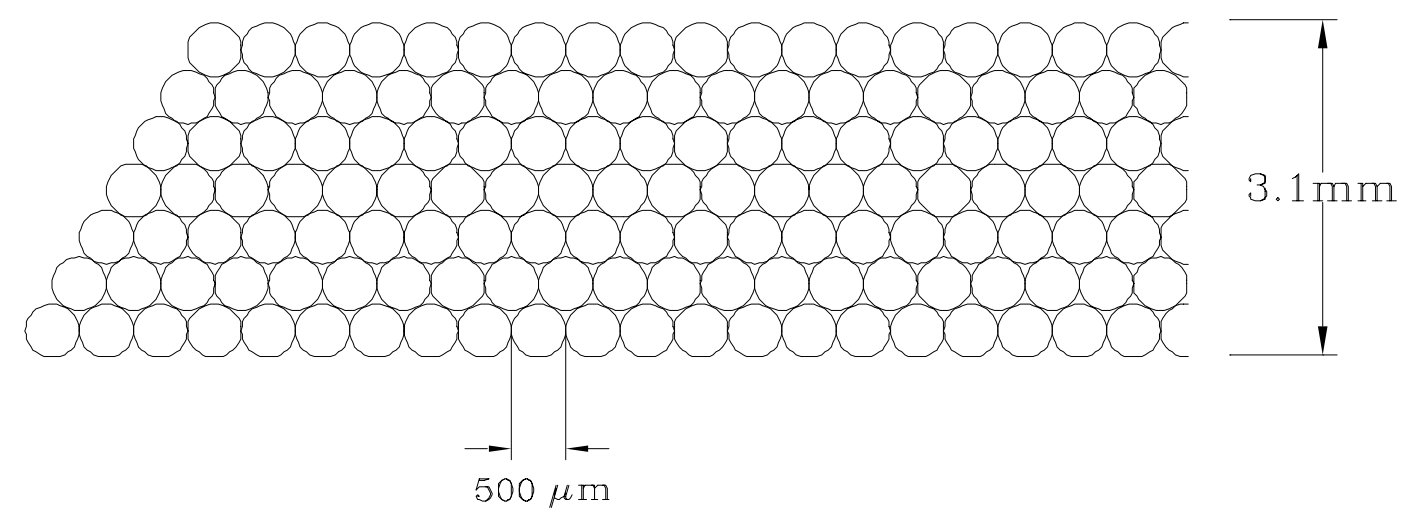

Figure 3 


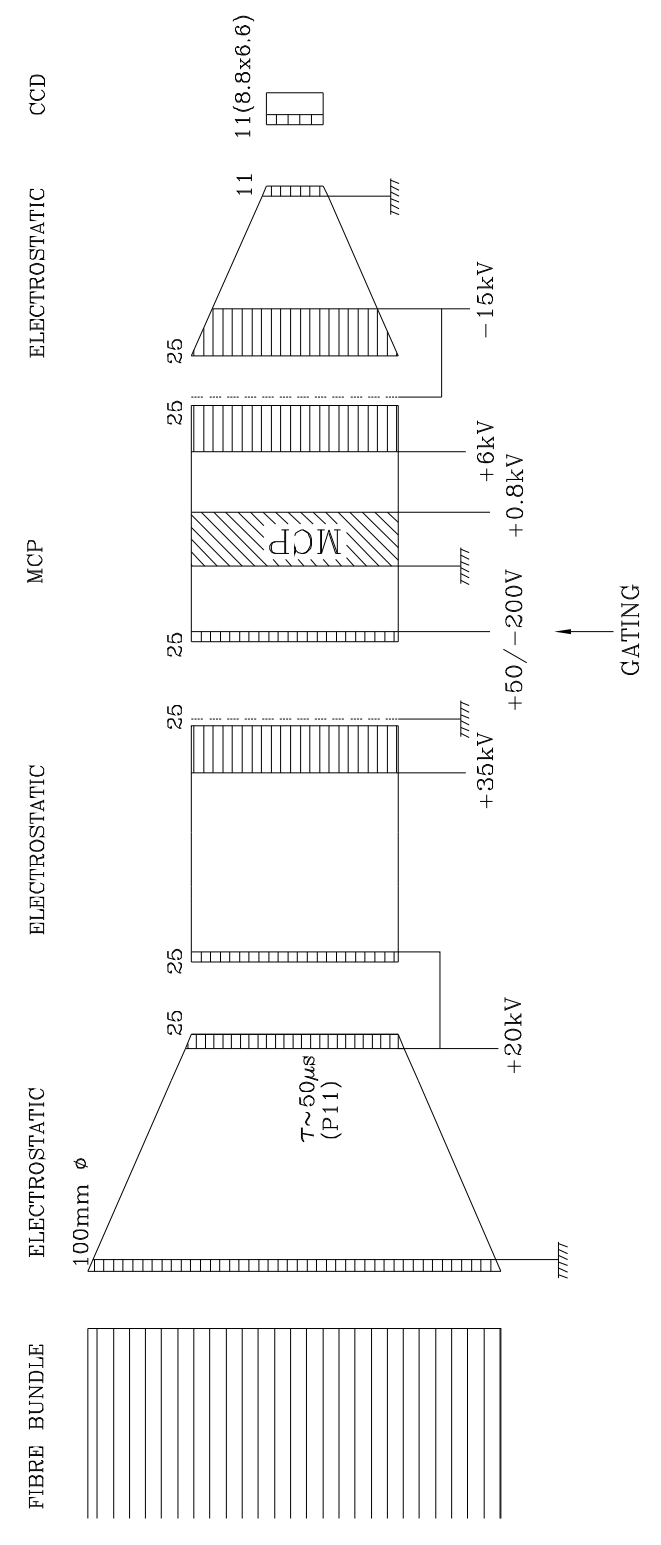

Figure 4 


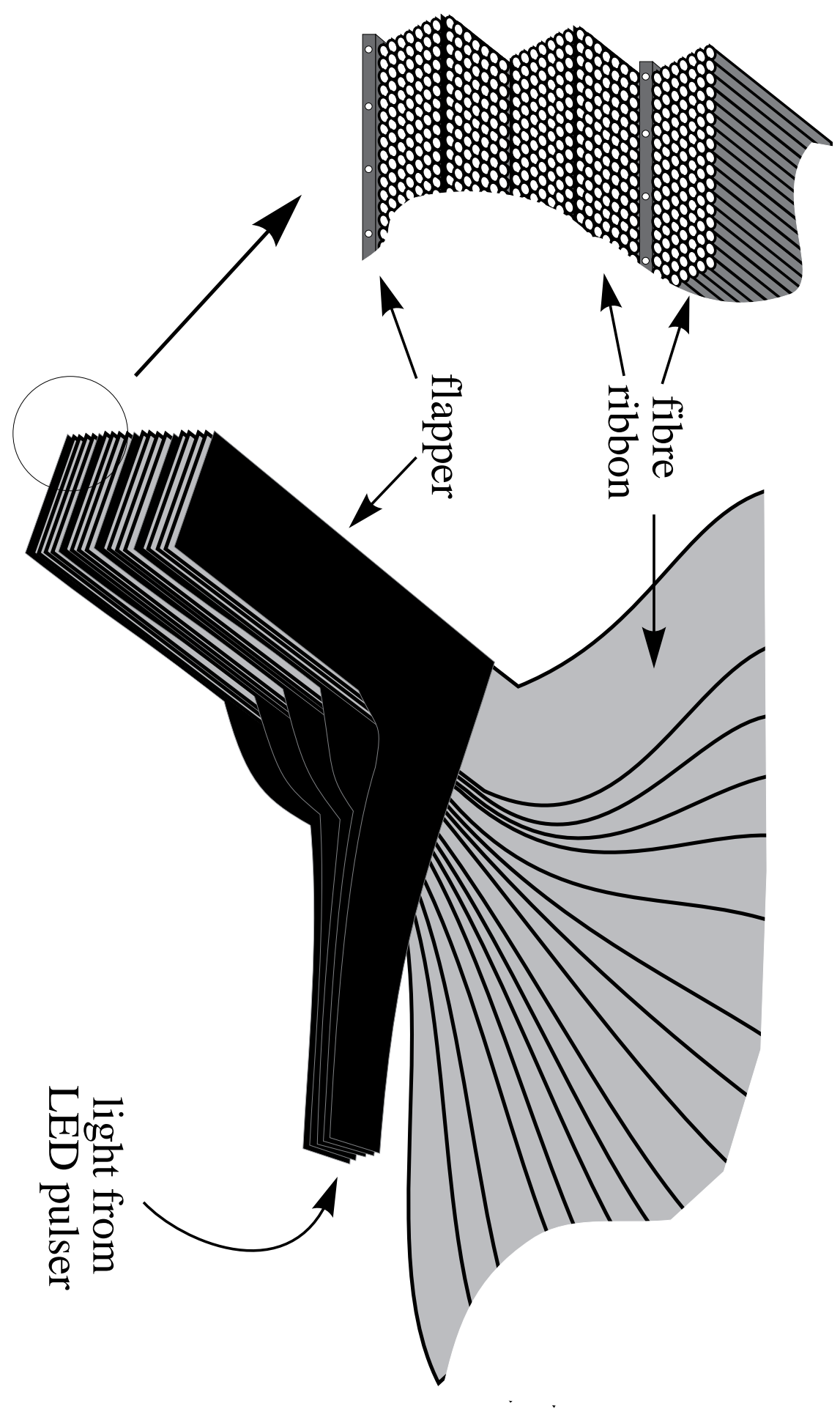

Figure 5 
9 FIDUCIAL FIBRES PER FLAPPER

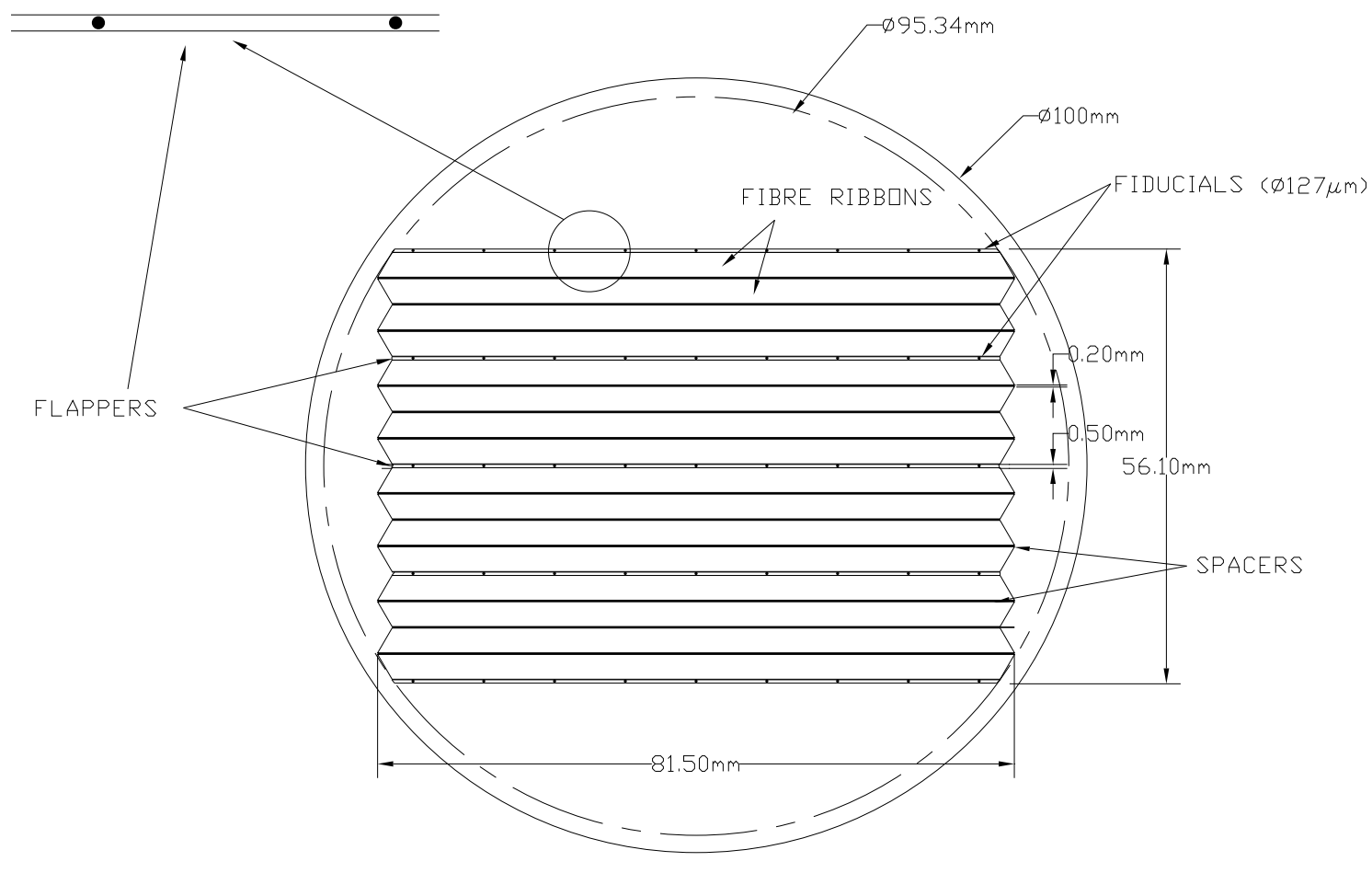

Figure 6a 


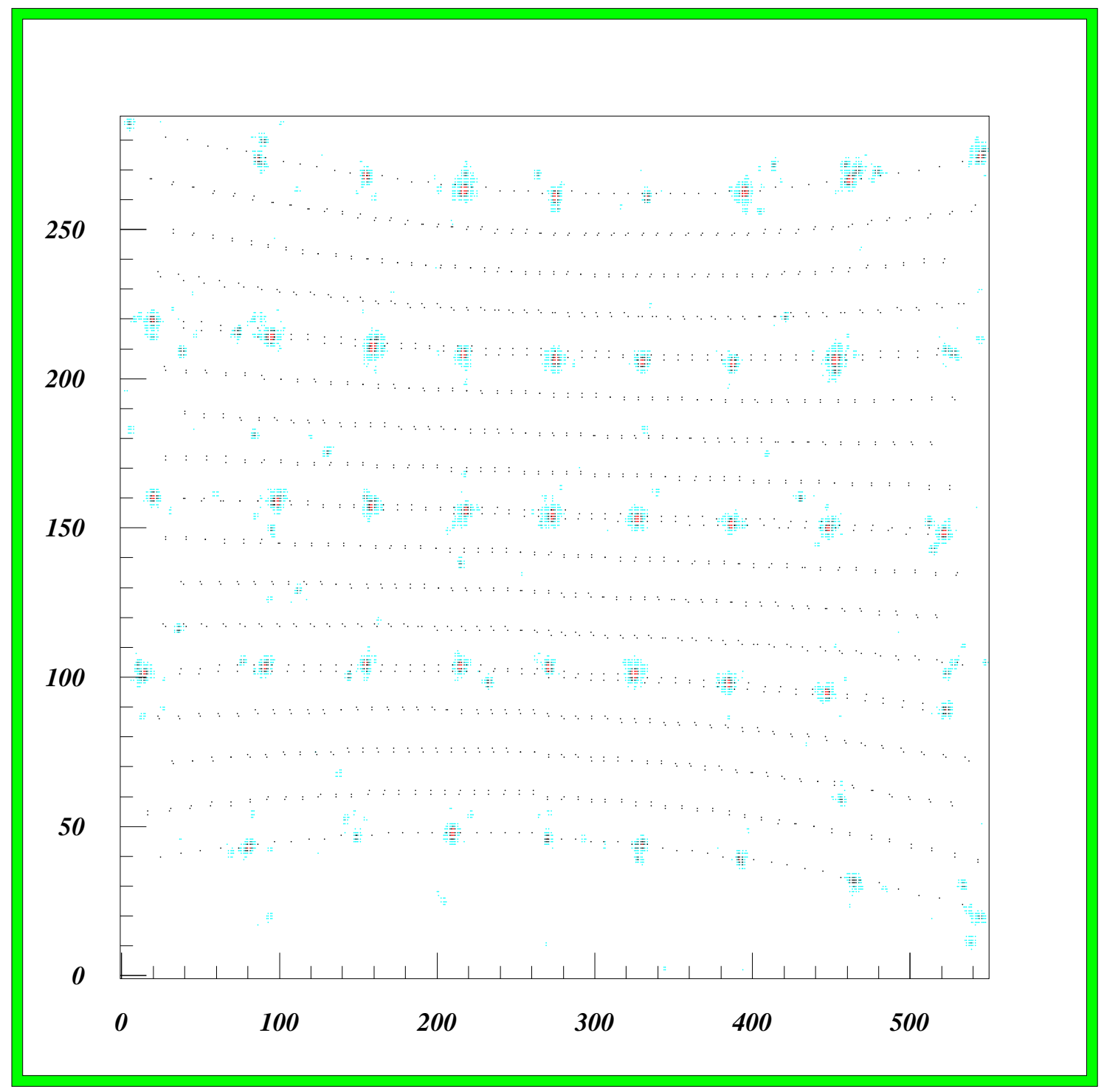

Figure $6 \mathrm{~b}$ 


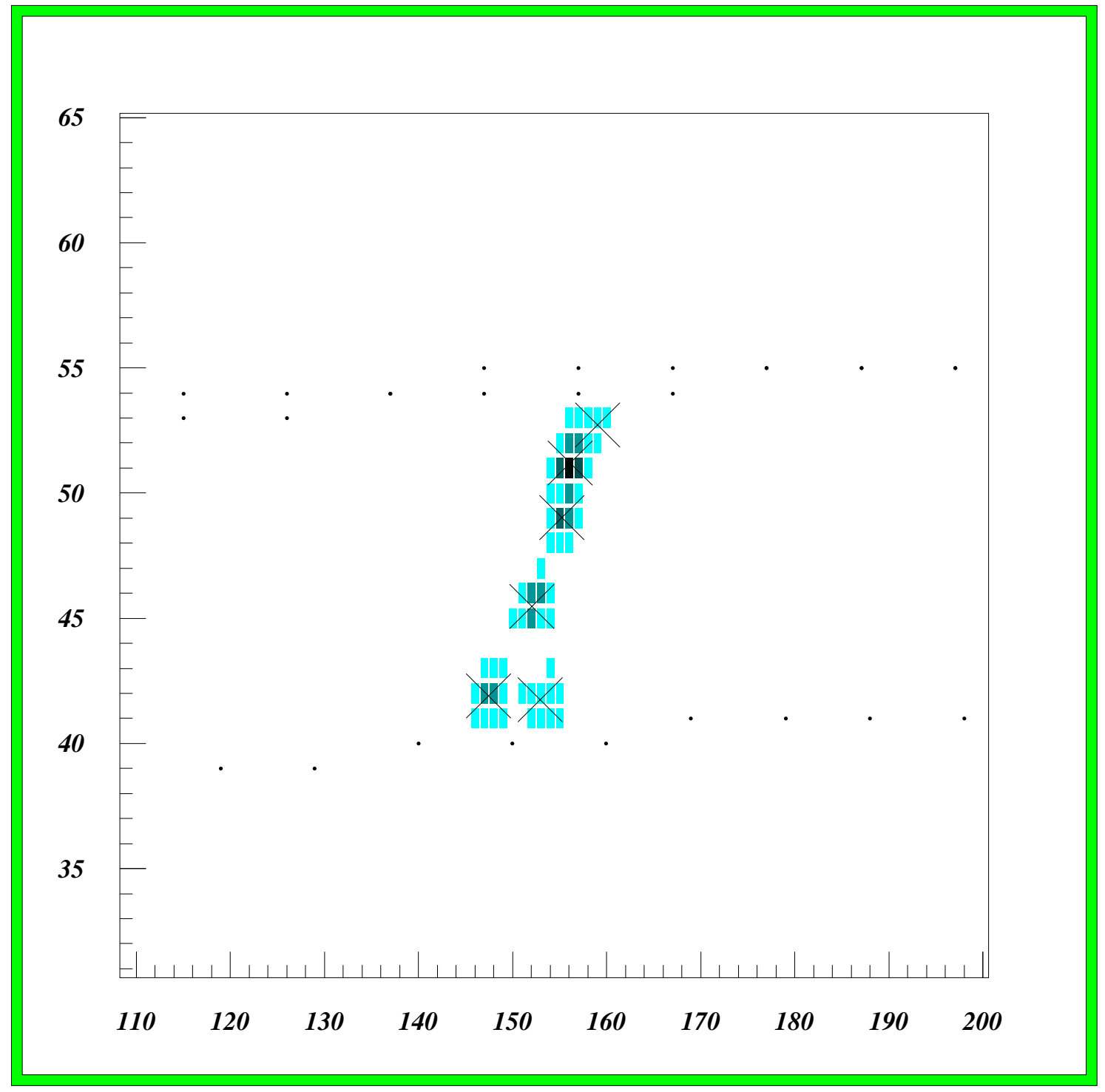

Figure 7 


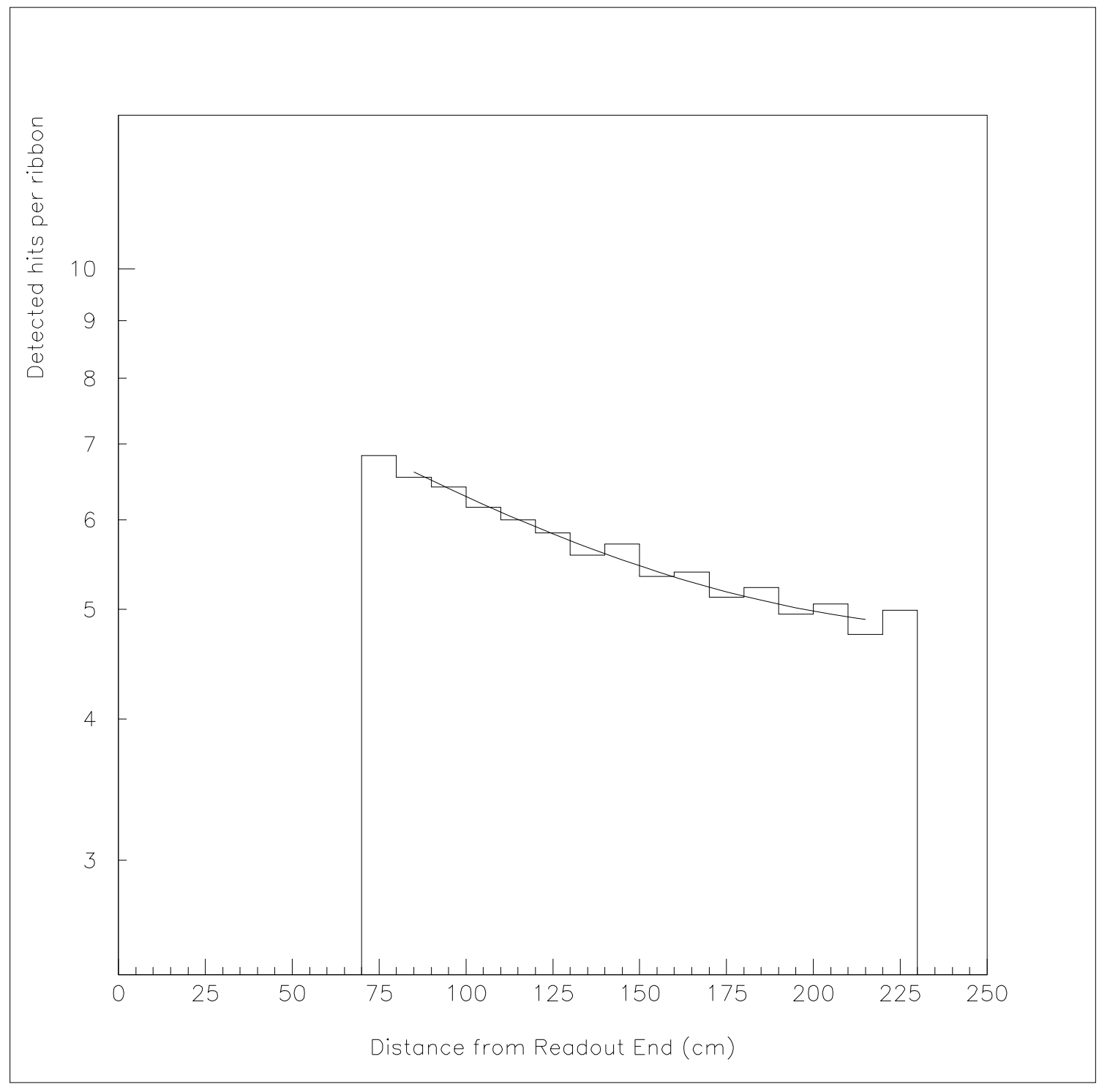

Figure 8 